\title{
On the singular sector of the Hermitian random matrix model in the large $N$ limit
}

\author{
B. Konopelchenko ${ }^{1}$, L. Martínez Alonso ${ }^{2}$ and E. Medina ${ }^{3}$ \\ ${ }^{1}$ Dipartimento di Fisica, Universitá del Salento and Sezione INFN \\ 73100 Lecce, Italy \\ 2 Departamento de Física Teórica II, Universidad Complutense \\ E28040 Madrid, Spain \\ ${ }^{3}$ Departamento de Matemáticas, Universidad de Cádiz \\ E11510 Puerto Real, Cádiz, Spain
}

January 3, 2014

\begin{abstract}
The singular sector of zero genus case for the Hermitian random matrix model in the large $N$ limit is analyzed. It is proved that the singular sector of the hodograph solutions for the underlying dispersionless Toda hierarchy and the singular sector of the 1-layer Benney (classical long wave equation) hierarchy are deeply connected. This property is due to the fact that the hodograph equations for both hierarchies describe the critical points of solutions of Euler-Poisson-Darboux equations $E(a, a)$, with $a=-1 / 2$ for the dToda hierarchy and $a=1 / 2$ for the 1-layer Benney hierarchy.
\end{abstract}

Key words: Integrable systems. Hodograph equations. Random matrix models. Euler-Poisson-Darboux equation.

PACS number: 02.30.Ik. 


\section{Introduction}

The Hermitian matrix $(H)$ model is nowadays the paradigmatic model in the theory of random matrices ( see e.g. [1, 2]). The partition function for this model is

$$
Z_{N}=\int \mathrm{d} H \exp \left(-\frac{N}{T} \operatorname{tr}\left(\sum_{k \geq 1} t_{k} H^{k}\right)\right),
$$

where $T>0$ is a real parameter which represents the temperature and the integration is performed on the space of $N \times N$ Hermitian matrices. The large $N$ limit of the $H$ model is of particular interest since it exhibits many important universality properties. It turns out that most of the applications of the $H$ model $[1,2]$ arise after regularizing the large $N$-limit solutions at their singular points (double-scaling limit method). In this sense the analysis and characterization of the singular sector of the $H$ model is of great relevance.

The simplest situation corresponds to the so called zero genus case in which the support of the eigenvalue density reduces to a single interval $\left[\beta_{1}, \beta_{2}\right]$ as $N \rightarrow \infty$. Moreover, it is well-known ( see e.g. $[3,4])$ that the endpoints $\boldsymbol{\beta}:=\left(\beta_{1}, \beta_{2}\right)$ evolve with the temperature $T$ and the coupling constants $t_{k}$ according to the dispersionless Toda (dToda) hierarchy . The first members of this hierarchy are the dToda equation $v_{T T}=(\log v)_{t_{1} t_{1}}$ or equivalently the dToda system

$$
u_{t_{1}}=-v_{T}, \quad v_{t_{1}}=-v u_{T}
$$

and the 1-layer Benney $(B)$ system

$$
u_{t_{2}}=2\left(u u_{t_{1}}+v_{t_{1}}\right), \quad v_{t_{2}}=2(u v)_{t_{1}} .
$$

These systems represent themselves two distinguished examples of integrable 2-component hydrodynamical type systems (see e.g. [5]). The dToda equation is the 1+1-dimensional version of the Boyer-Finley equation from the general relativity [6]. It also arises in various problems of fluid mechanics (see e.g. $[7,8,9]$ ). The $B$ system describes long waves in shallow water with free surface in a gravitational field [10]. It represents the dispersionless limit of the celebrated nonlinear Schrödinger equation [11]. Recently, the $B$ system became a crucial ingredient in the analysis of the universality of critical behaviour for nonlinear equations [12]. In general, the $B$ system (3) is an excellent laboratory for study properties of integrable hydrodynamical type systems.

Thus, the analysis of the singular sector for the $H$ model in the large $N$ limit reduces to the corresponding analysis for the dToda hierarchy. Recently the authors [13] have provided a method for studying the hodograph solutions and their singular sectors (gradient catastrophe points) of the family of coupled $\mathrm{KdV}$ hierarchies $\operatorname{dcKdV}_{m}(m \geq 1)$, where the case $m=2$ corresponds to the $B$ hierarchy. The study of these singular sectors was already initiated in [14]. In the method of [13] the hodograph solutions are described by the critical points of scalar functions that satisfy Euler-Poisson-Darboux (EPD) equations [15]. This property simplifies drastically the analysis and classification of the singular sectors .

In the present paper we show that the results of [13] can be extended to the dToda hierarchy thus providing us with complete analysis of the singular sector of $H$ model for the zero genus case. Moreover, we demonstrate that there is deep similarity between the properties of the sets of the hodograph solutions for the dToda and $B$ hierarchies.

Our main observation is that in both cases the hodograph solutions represent the critical points

$$
\frac{\partial W}{\partial \beta_{i}}=0, \quad i=1,2
$$


of a scalar function $W$ which depends linearly on the coordinates $\boldsymbol{t}=\left(t_{0}, t_{1}, t_{2}, \ldots\right)$, where $\boldsymbol{t}$ is the set of coupling constants for the $H$ model $\left(t_{0}=T\right)$ and the set of flow parameters in the case of the $B$ hierarchy. Moreover, these functions satisfy the Euler-Poisson-Darboux equation $E(a, a)$ $[15]$

$$
\left(\beta_{1}-\beta_{2}\right) \frac{\partial^{2} W}{\partial \beta_{1} \partial \beta_{2}}=a\left(\frac{\partial W}{\partial \beta_{1}}-\frac{\partial W}{\partial \beta_{2}}\right), \quad a=\left\{\begin{aligned}
\frac{1}{2} & \text { for } W_{B} \\
-\frac{1}{2} & \text { for } W_{H} .
\end{aligned}\right.
$$

Both functions are related according to a transformation [15] which maps solutions of $E(-1 / 2,-1 / 2)$ into solutions $E(1 / 2,1 / 2)$

$$
W_{B}=4 \frac{\partial^{2} W_{H}}{\partial \beta_{1} \partial \beta_{2}}-t_{H, 1}
$$

where the parameters of both models are identified according to

$$
t_{B, n}=(n+2) t_{H, n+2}, \quad n \geq 0 .
$$

The equation (5) and its multidimensional version are well known for a long time in the classical geometry [15]. Its relevance to the theory of Whitham equations has been demonstrated recently in the papers [16]-[17]. The observation made in [13] that the hodograph equations for the $B$ system (3) have the form (4)-(5) seems to be new, though results close to this have been provided in the papers $[12,18]$.

The paper is organized as follows. In Section 2 we show how the hodograph equations of the $B$ system and the $H$ models are the equations for the critical points of certain solutions of EulerPoisson-Darboux equations. In Section 3 we use the Euler-Poisson-Darboux equations to formulate a common description of the singular sectors for theses hodograph equations. Our results are summarized into three Propositions which exhibit the deep connection arising between the singular sectors of both models. Moreover, the corresponding singular classes are also characterized in terms of the behaviour near $\lambda=\beta_{i}(i=1,2)$ of the $S$-function ( $B$ system) and the eigenvalue density $\rho$ ( $H$ model). Section 4 is devoted to a method for the explicit determination of singular classes by means of constraints for the $\boldsymbol{t}$-parameters. Finally, some illustrative examples are provided.

\section{Hodograph equations and the Euler-Poisson-Darboux equations}

\section{B hierarchy}

The $B$ system (3) is a member of a dispersionless integrable hierarchy which describe deformations of the curve (see e.g. [14, 19]).

$$
p^{2}=\left(\lambda-\beta_{1}\right)\left(\lambda-\beta_{2}\right)
$$

The flows

$$
\boldsymbol{\beta}(\boldsymbol{t}), \quad \boldsymbol{t}=\left(x:=t_{0}, t_{1}, t_{2}, \ldots\right)
$$

are characterized by the following condition: There exists a family of functions $S(\lambda, \boldsymbol{t}, \boldsymbol{\beta})$ satisfying

$$
\frac{\partial S(\lambda, \boldsymbol{t}, \boldsymbol{\beta}(\boldsymbol{t}))}{\partial t_{n}}=\Omega_{n}(\lambda, \boldsymbol{\beta}(\boldsymbol{t})), \quad n \geq 0 .
$$


where

$$
\Omega_{n}(\lambda, \boldsymbol{\beta})=\left(\frac{\lambda^{n+1}}{\sqrt{\left(\lambda-\beta_{1}\right)\left(\lambda-\beta_{2}\right)}}\right)_{\oplus} \sqrt{\left(\lambda-\beta_{1}\right)\left(\lambda-\beta_{2}\right)} .
$$

Functions $S$ which satisfy (7) are referred to as action functions in the theory of dispersionless integrable systems (see e.g. [20]). Notice that for $n=0$ Eq.(7) reads

$$
p=\frac{\partial S}{\partial x}
$$

so that the system (7) is equivalent to

$$
\frac{\partial p}{\partial t_{n}}=\partial_{x} \Omega_{n}
$$

and, in terms of $\boldsymbol{\beta}$, it can be rewritten in the hydrodynamical form

$$
\frac{\partial \beta_{i}}{\partial t_{n}}=\left.\left(\Omega_{n}(\lambda, \boldsymbol{\beta})\right)_{\oplus}\right|_{\lambda=\beta_{i}} \partial_{x} \beta_{i}, \quad i=1,2 .
$$

The $t_{1}$-flow of this hierarchy is the $B$ system (3) $\left(t:=t_{1}\right)$, which in terms of $\boldsymbol{\beta}$ reads

$$
\left\{\begin{array}{l}
\partial_{t} \beta_{1}=\frac{1}{2}\left(3 \beta_{1}+\beta_{2}\right) \beta_{1 x} \\
\partial_{t} \beta_{2}=\frac{1}{2}\left(3 \beta_{2}+\beta_{1}\right) \beta_{2 x}
\end{array}\right.
$$

It was proved in [13] that the system (4) for the function

$$
W_{B}(\boldsymbol{t}, \boldsymbol{\beta}):=\oint_{\gamma} \frac{\mathrm{d} \lambda}{2 i \pi} \frac{\lambda V_{B}(\lambda, \boldsymbol{t})}{\sqrt{\left(\lambda-\beta_{1}\right)\left(\lambda-\beta_{2}\right)}},
$$

where

$$
V_{B}(\lambda, \boldsymbol{t})=\sum_{n \geq 0} \lambda^{n} t_{n}
$$

is a system of hodograph equations for the $B$ hierarchy. Moreover, the action function for the corresponding solutions is given by

$$
S(\lambda, \boldsymbol{t}, \boldsymbol{\beta})=\sum_{n \geq 0} t_{n} \Omega_{n}(\lambda, \boldsymbol{\beta})=h_{B}(\lambda, \boldsymbol{t}, \boldsymbol{\beta}) \sqrt{\left(\lambda-\beta_{1}\right)\left(\lambda-\beta_{2}\right)} .
$$

where

$$
h_{B}(\lambda, \boldsymbol{t}, \boldsymbol{\beta}):=\left(\frac{\lambda V_{B}(\lambda, \boldsymbol{t})}{\sqrt{\left(\lambda-\beta_{1}\right)\left(\lambda-\beta_{2}\right)}}\right)_{\oplus} .
$$

Obviously, $W_{B}$ satisfies the Euler-Poisson-Darboux equation $E\left(\frac{1}{2}, \frac{1}{2}\right)$. Written explicitly, $W_{B}$ represents itself the series

$$
\begin{aligned}
W_{B} & =\frac{x}{2}\left(\beta_{1}+\beta_{2}\right)+\frac{t_{1}}{8}\left(3 \beta_{1}^{2}+2 \beta_{1} \beta_{2}+3 \beta_{2}^{2}\right)+\frac{t_{2}}{16}\left(5 \beta_{1}^{3}+3 \beta_{1}^{2} \beta_{2}+3 \beta_{1} \beta_{2}^{2}+5 \beta_{2}^{3}\right) \\
& +\frac{t_{3}}{128}\left(35 \beta_{1}^{4}+20 \beta_{1}^{3} \beta_{2}+18 \beta_{1}^{2} \beta_{2}^{2}+20 \beta_{1} \beta_{2}^{3}+35 \beta_{2}^{4}\right)+\cdots
\end{aligned}
$$


The hodograph equations (4) with $t_{n}=0$ for $n \geq 4$ take the form

$$
\left\{\begin{array}{l}
8 x+4 t_{1}\left(3 \beta_{1}+\beta_{2}\right)+3 t_{2}\left(5 \beta_{1}^{2}+2 \beta_{1} \beta_{2}+\beta_{2}^{2}\right)+\frac{t_{3}}{8}\left(140 \beta_{1}^{3}+60 \beta_{1}^{2} \beta_{2}+36 \beta_{1} \beta_{2}^{2}+20 \beta_{2}^{2}\right)=0 \\
8 x+4 t_{1}\left(\beta_{1}+3 \beta_{2}\right)+3 t_{2}\left(\beta_{1}^{2}+2 \beta_{1} \beta_{2}+5 \beta_{2}^{2}\right)+\frac{t_{3}}{8}\left(140 \beta_{2}^{3}+60 \beta_{2}^{2} \beta_{1}+36 \beta_{2} \beta_{1}^{2}+20 \beta_{1}^{2}\right)=0 .
\end{array}\right.
$$

Detailed analysis of equations (15) will be performed in section 4 .

\section{dToda hierarchy}

It is known (see e.g. [1]) that as $N \rightarrow \infty$ the asymptotic density of eigenvalues $\left\{\lambda_{1}, \ldots, \lambda_{N}\right\}$ of the $H$ model in the zero genus case concentrates on a single interval $\left[\beta_{1}, \beta_{2}\right]$ and is given by [1]

$$
\rho(\lambda, \boldsymbol{t}, \boldsymbol{\beta}):=\frac{h_{H}(\lambda, \boldsymbol{t}, \boldsymbol{\beta})}{2 \pi i T} \sqrt{\left(\lambda-\beta_{1}\right)\left(\lambda-\beta_{2}\right)},
$$

where

$$
h_{H}(\lambda, \boldsymbol{t}, \boldsymbol{\beta}):=\left(\frac{V_{H, \lambda}(\lambda, \boldsymbol{t})}{\sqrt{\left(\lambda-\beta_{1}\right)\left(\lambda-\beta_{2}\right)}}\right)_{\oplus} .
$$

Here $\oplus$ denotes the projection on strictly positive powers of $\lambda$. Moreover, the endpoints $\beta_{1}$ and $\beta_{2}$ of the eigenvalue support are determined by the equations

$$
\oint_{\gamma} \frac{\mathrm{d} \lambda}{2 i \pi} \frac{\partial_{\lambda} V_{H}(\lambda, \boldsymbol{t})}{\sqrt{\left(\lambda-\beta_{1}\right)\left(\lambda-\beta_{2}\right)}}=0, \quad \oint_{\gamma} \frac{\mathrm{d} \lambda}{2 i \pi} \frac{\lambda \partial_{\lambda} V_{H}(\lambda, \boldsymbol{t})}{\sqrt{\left(\lambda-\beta_{1}\right)\left(\lambda-\beta_{2}\right)}}=2 T
$$

where

$$
V_{H}(\lambda, \boldsymbol{t}):=\sum_{n \geq 1} \lambda^{n} t_{n}
$$

and $\gamma$ denotes a large positively oriented circle $|\lambda|=R$. It is immediate to see that these equations are equivalent to the system (4) for the critical points of the function

$$
W_{H}(\boldsymbol{t}, \boldsymbol{\beta}):=T\left(\beta_{1}+\beta_{2}\right)+\oint_{\gamma} \frac{\mathrm{d} \lambda}{2 i \pi} \sqrt{\left(\lambda-\beta_{1}\right)\left(\lambda-\beta_{2}\right)} \partial_{\lambda} V_{H}(\lambda, \boldsymbol{t}),
$$

which satisfies the Euler-Poisson-Darboux equation $E\left(-\frac{1}{2},-\frac{1}{2}\right)$. It is also easy to check that the functions $W_{B}$ and $W_{H}$ are related according to

$$
W_{B}=4 \frac{\partial^{2} W_{H}}{\partial \beta_{1} \partial \beta_{2}}-t_{H, 1}, \quad t_{B, n}=(n+2) t_{H, n+2}, \quad n \geq 0 .
$$

The first terms of the function $W_{H}$ are

$$
\begin{aligned}
W_{H} & =T\left(\beta_{1}+\beta_{2}\right)-\frac{t_{1}}{8}\left(\beta_{1}-\beta_{2}\right)^{2}-\frac{t_{2}}{8}\left(\beta_{1}+\beta_{2}\right)\left(\beta_{1}-\beta_{2}\right)^{2}-\frac{3 t_{3}}{128}\left(5 \beta_{1}^{2}+6 \beta_{2} \beta_{1}+5 \beta_{2}^{2}\right)\left(\beta_{1}-\beta_{2}\right)^{2} \\
& -\frac{t_{4}}{64}\left(7 \beta_{1}^{3}+9 \beta_{2} \beta_{1}^{2}+9 \beta_{2}^{2} \beta_{1}+7 \beta_{2}^{3}\right)\left(\beta_{1}-\beta_{2}\right)^{2}+\cdots,
\end{aligned}
$$


The corresponding equations (4) for $t_{n}=0, \forall n \geq 4$ take the form

$$
\left\{\begin{array}{l}
T+\frac{t_{1}}{4}\left(\beta_{2}-\beta_{1}\right)+\frac{t_{2}}{8}\left(-3 \beta_{1}^{2}+2 \beta_{2} \beta_{1}+\beta_{2}^{2}\right)-\frac{3 t_{3}}{32}\left(5 \beta_{1}^{3}-3 \beta_{2} \beta_{1}^{2}-\beta_{2}^{2} \beta_{1}-\beta_{2}^{3}\right)=0 \\
T+\frac{t_{1}}{4}\left(\beta_{1}-\beta_{2}\right)+\frac{t_{2}}{8}\left(\beta_{1}^{2}+2 \beta_{2} \beta_{1}-3 \beta_{2}^{2}\right)+\frac{3 t_{3}}{32}\left(\beta_{1}^{3}+\beta_{2} \beta_{1}^{2}+3 \beta_{2}^{2} \beta_{1}-5 \beta_{2}^{3}\right)=0 .
\end{array}\right.
$$

It is known that the hodograph system (16) provides solutions of the dispersionless Toda hierarchy (see e.g. $[3,4])$. Indeed, for "times" $T$ and $t_{1}$ one gets

$$
\left\{\begin{array}{l}
\partial_{t_{1}} \beta_{1}=-\frac{1}{4}\left(\beta_{1}-\beta_{2}\right) \beta_{1 T} \\
\partial_{t_{1}} \beta_{2}=\frac{1}{4}\left(\beta_{1}-\beta_{2}\right) \beta_{2 T}
\end{array}\right.
$$

which in terms of variables

$$
u=\frac{1}{2}\left(\beta_{1}+\beta_{2}\right), \quad v=\frac{1}{16}\left(\beta_{1}-\beta_{2}\right)^{2},
$$

becomes the dToda system (2). Moreover, for times $t_{1}, t_{2}$ the hodograph equations (16) imply the Benney system (3). If we use the dependent variables $(u, v)$ and introduce the function

$$
f(\boldsymbol{t}, u, v):=W_{H}(\boldsymbol{t}, u, v)-2 T u+2 t_{1} v+4 t_{2} u v,
$$

then the hodograph equations read

$$
\left\{\begin{array}{l}
2 T-4 v t_{2}+\partial_{u} f=0 \\
-2 t_{1}-4 u t_{2}+\partial_{v} f=0
\end{array}\right.
$$

which, under trivial rescalings and the substitution $(u, v) \mapsto(v, u)$, coincides with the hyperbolic version of hodograph system (2.11) of [12]. In particular, from the EPD equation (5) it follows easily that

$$
\frac{\partial^{2} W_{H}}{\partial u^{2}}-v \frac{\partial^{2} W_{H}}{\partial v^{2}}=\frac{\partial^{2} f}{\partial u^{2}}-v \frac{\partial^{2} f}{\partial v^{2}}=0
$$

\section{Characterization of singular sectors}

Using (4) and (5) we may now analyze the structure of singular sectors of the $B$ system and the $H$ model in a unified way. Thus, let us denote by $\mathcal{M}$ the set of solutions $(\boldsymbol{t}, \boldsymbol{\beta})\left(\beta_{1} \neq \beta_{2}\right)$ of the hodograph equations (4). There is a partition of $\mathcal{M}$ into a regular and a singular sector

$$
\mathcal{M}=\mathcal{M}^{\mathrm{reg}} \cup \mathcal{M}^{\operatorname{sing}}
$$

such that given $(\boldsymbol{t}, \boldsymbol{\beta}) \in \mathcal{M}$

$$
(\boldsymbol{t}, \boldsymbol{\beta}) \in \mathcal{M}^{\mathrm{reg}} \text { if } \operatorname{det}\left(\frac{\partial^{2} W(\boldsymbol{t}, \boldsymbol{\beta})}{\partial \beta_{i} \partial \beta_{j}}\right) \neq 0, \quad(\boldsymbol{t}, \boldsymbol{\beta}) \in \mathcal{M}^{\operatorname{sing}} \text { if } \operatorname{det}\left(\frac{\partial^{2} W(\boldsymbol{t}, \boldsymbol{\beta})}{\partial \beta_{i} \partial \beta_{j}}\right)=0
$$


The elements of $\mathcal{M}^{\text {reg }}$, correspond to the case when the system (4) is uniquely solvable and hence, it defines a unique solution $\boldsymbol{\beta}(\boldsymbol{t})$. The singular sector $\mathcal{M}^{\text {sing }}$ contains the degenerate critical points of the function $W$ on which the implicit solutions $\boldsymbol{\beta}(\boldsymbol{t})$ of the hodograph equations exhibit gradient catastrophe behaviour.

The Euler-Poisson-Darboux equation is of great help to analyze the structure of $\mathcal{M}^{\text {sing }}$. Indeed, if $(\boldsymbol{t}, \boldsymbol{\beta}) \in \mathcal{M}$, as a consequence of (5) it is clear that

$$
\frac{\partial^{2} W}{\partial \beta_{1} \partial \beta_{2}}=0
$$

Consequently

$$
\operatorname{det}\left(\frac{\partial^{2} W}{\partial \beta_{i} \partial \beta_{j}}\right)=\frac{\partial^{2} W}{\partial \beta_{1}^{2}} \cdot \frac{\partial^{2} W}{\partial \beta_{2}^{2}}
$$

Thus, we have

Proposition 1. Given $(\boldsymbol{t}, \boldsymbol{\beta}) \in \mathcal{M}$ then

1. $(\boldsymbol{t}, \boldsymbol{\beta}) \in \mathcal{M}^{\text {reg }}$ if and only if $\frac{\partial^{2} W}{\partial \beta_{1}^{2}} \neq 0$ and $\frac{\partial^{2} W}{\partial \beta_{2}^{2}} \neq 0$.

2. $(\boldsymbol{t}, \boldsymbol{\beta}) \in \mathcal{M}^{\text {sing }}$ if and only at least one of the derivatives $\frac{\partial^{2} W}{\partial \beta_{1}^{2}}, \frac{\partial^{2} W}{\partial \beta_{2}^{2}}$, vanishes.

Furthermore, using (5) it follows at once that at any point $(\boldsymbol{t}, \boldsymbol{\beta}) \in \mathcal{M}$ all mixed derivatives $\partial_{\beta_{1}}^{i} \partial_{\beta_{2}}^{j} W$ can be expressed in terms of linear combination of diagonal derivatives $\partial_{\beta_{1}}^{n} W$ and $\partial_{\beta_{2}}^{m} W$. Let us now define $\mathcal{M}_{n_{1}, n_{2}}^{\text {sing }}$ as the set of points $(\boldsymbol{t}, \boldsymbol{\beta}) \in \mathcal{M}$ such that

$$
\frac{\partial^{n_{i}+2} W}{\partial \beta_{i}^{n_{i}+2}} \neq 0 ; \quad \frac{\partial^{k} W}{\partial \beta_{i}^{k}}=0, \quad \forall 1 \leq k \leq n_{i}+1, \quad(i=1,2) .
$$

It is clear that

$$
\mathcal{M}_{n_{1}, n_{2}}^{\operatorname{sing}} \bigcap \mathcal{M}_{n_{1}^{\prime}, n_{2}^{\prime}}^{\text {sing }}=\emptyset, \text { for }\left(n_{1}, n_{2}\right) \neq\left(n_{1}^{\prime}, n_{2}^{\prime}\right)
$$

Then, it follows at once that

Proposition 2. The singular sector satisfies

$$
\mathcal{M}^{\text {sing }}=\bigcup_{n_{1}+n_{2} \geq 1} \mathcal{M}_{n_{1}, n_{2}}^{\text {sing }} .
$$

According to (18) and using (5) we have

$$
W_{B}=-t_{H, 1}-\frac{2}{\beta_{1}-\beta_{2}}\left(\frac{\partial W_{H}}{\partial \beta_{1}}-\frac{\partial W_{H}}{\partial \beta_{2}}\right), \quad t_{B, n}=(n+2) t_{H, n+2}, \quad n \geq 0 .
$$

It is then immediate to conclude that

Proposition 3. The singular sectors of the $H$ model and the Benney system, with $t_{B, n}=(n+2) t_{H, n+2}$ for $n \geq 0$, satisfy

$$
\left\{\begin{array}{l}
\mathcal{M}_{H, 1,1}^{\text {sing }} \subset \mathcal{M}_{B}^{\text {reg }}, \\
\mathcal{M}_{H, n_{1}+1, n_{2}+1}^{\text {sing }} \subset \mathcal{M}_{B, n_{1}, n_{2}}^{\text {sing }} \text { for } n_{1} \geq 1, n_{2} \geq 1 .
\end{array}\right.
$$




\section{Singular sectors in the $H$ model}

In the applications of the $H$ model in quantum gravity [1] the singular sectors are described in terms of the behaviour of the eigenvalue density $\rho(\lambda)$ at the endpoints $\left(\beta_{1}, \beta_{2}\right)$ of its support. We will now show how this description derives in a natural way from the classification (27) based on

the subsets $\mathcal{M}_{H, n_{1}, n_{2}}^{\text {sing }}$ of the singular sector . To this end let us consider the derivative $\partial_{\beta_{1}}^{k+1} W_{H}$ with $k \geq 2$, it is obviously proportional to the integral

$\oint_{\gamma} \frac{\mathrm{d} \lambda}{2 i \pi}\left(\lambda-\beta_{2}\right) \frac{V_{H, \lambda} / \sqrt{\left(\lambda-\beta_{1}\right)\left(\lambda-\beta_{2}\right)}}{\left(\lambda-\beta_{1}\right)^{k}}=\oint_{\gamma} \frac{\mathrm{d} \lambda}{2 i \pi} \frac{\left(\lambda-\beta_{2}\right) h_{H}(\lambda)}{\left(\lambda-\beta_{1}\right)^{k}}=\left.\frac{\partial_{\lambda}^{k-1}}{(k-1) !}\left(\left(\lambda-\beta_{2}\right) h_{H}(\lambda)\right)\right|_{\lambda=\beta_{1}}$,

and a similar result follows for the derivatives $\partial_{\beta_{2}}^{k+1} W$ with $k \geq 2$. As a consequence we have

Proposition 4. A point $(\boldsymbol{t}, \boldsymbol{\beta}) \in \mathcal{M}_{H}$ belongs to the singularity class $\mathcal{M}_{H, n_{1}, n_{2}}^{\text {sing }}$ if and only if

$$
\rho(\lambda, \boldsymbol{t}, \boldsymbol{\beta}) \sim\left(\lambda-\beta_{i}\right)^{\frac{2 n_{i}+1}{2}} \quad \text { as } \lambda \rightarrow \beta_{i}, \quad(i=1,2)
$$

This property is crucial to establish the relationship between the regularized singularity sectors of the $H$ model and the minimal conformal models $(p, q)$ with $q=2[1]$.

\section{Singular sectors in the $B$ system}

In analogy with the $H$ model we may characterize the classes $\mathcal{M}_{B, n_{1}, n_{2}}^{\operatorname{sing}}$ of the singular sector of the $B$ system in terms of the behaviour of $S(\lambda)$ at $\lambda=\beta_{i}(i=1,2)$. Indeed the derivative $\partial_{\beta_{1}}^{k+1} W_{B}$ with $k \geq 1$ is proportional to the integral

$\oint_{\gamma} \frac{\mathrm{d} \lambda}{2 i \pi}\left(\lambda-\beta_{2}\right) \frac{\lambda V_{B} / \sqrt{\left(\lambda-\beta_{1}\right)\left(\lambda-\beta_{2}\right)}}{\left(\lambda-\beta_{1}\right)^{k+1}}=\oint_{\gamma} \frac{\mathrm{d} \lambda}{2 i \pi} \frac{\left(\lambda-\beta_{2}\right) h_{B}(\lambda)}{\left(\lambda-\beta_{1}\right)^{k+1}}=\left.\frac{\partial_{\lambda}^{k}}{k !}\left(\left(\lambda-\beta_{2}\right) h_{B}(\lambda)\right)\right|_{\lambda=\beta_{1}}$,

and a similar result follows for the derivatives $\partial_{\beta_{2}}^{k+1} W_{B}$ with $k \geq 2$. As a consequence we have

Proposition 5. A point $(\boldsymbol{t}, \boldsymbol{\beta}) \in \mathcal{M}_{B}$ belongs to the singularity class $\mathcal{M}_{B, n_{1}, n_{2}}^{\text {sing }}$ if and only if

$$
S(\lambda, \boldsymbol{t}, \boldsymbol{\beta}) \sim\left(\lambda-\beta_{i}\right)^{\frac{2 n_{i}+3}{2}} \quad \text { as } \lambda \rightarrow \beta_{i}, \quad(i=1,2)
$$

This result shows the existing duality between the eigenvalue density $\rho$ of the $H$ model and the $S$ function of the $B$ system.

\section{Explicit determination of singular sectors in terms of constrains $f_{k}(\boldsymbol{t})=0$}

The singular classes $\mathcal{M}_{n_{1}, n_{2}}^{\text {sing }}$ can be determined by means of systems of $n_{1}+n_{2}$ constraints for the coordinates $\boldsymbol{t}$. To see this property notice that the points $(\boldsymbol{t}, \boldsymbol{\beta})$ of $\mathcal{M}_{n_{1}, n_{2}}^{\mathrm{sing}}$ are characterized by the equations

$$
\frac{\partial^{k} W}{\partial \beta_{i}^{k}}=0, \quad \forall 1 \leq k \leq n_{i}+1, \quad i=1,2
$$


and

$$
\frac{\partial^{n_{i}+2} W}{\partial \beta_{i}^{n_{i}+2}} \neq 0, \quad i=1,2 .
$$

Furthermore, the jacobian matrix of the the system of two equations

$$
\frac{\partial^{n_{i}+1} W}{\partial \beta_{i}^{n_{i}+1}}=0, \quad i=1,2
$$

is not singular as

$$
\Delta:=\left|\begin{array}{cc}
\frac{\partial^{n_{1}+2} W}{\partial \beta_{1}^{n_{1}+2}} & \frac{\partial^{n_{2}+2} W}{\partial \beta_{1} \partial \beta_{2}^{n_{2}+1}} \\
\frac{\partial^{n_{1}+2} W}{\partial \beta_{1}^{n_{1}+1} \partial \beta_{2}} & \frac{\partial^{n_{2}+2} W}{\partial \beta_{2}^{n_{2}+2}}
\end{array}\right| \neq 0 .
$$

Indeed, we notice that as a consequence of (5) the derivatives outside the diagonal of $\Delta$ are linear combinations of the derivatives $\left\{\partial_{\beta_{i}}^{k} W, 1 \leq k \leq n_{i}+1, i=1,2\right\}$, so that from (31)-(32) we have

$$
\Delta=\frac{\partial^{n_{1}+2} W}{\partial \beta_{1}^{n_{1}+2}} \cdot \frac{\partial^{n_{2}+2} W}{\partial \beta_{2}^{n_{2}+2}} \neq 0 .
$$

Therefore, one can solve (33) and get a solution $\boldsymbol{\beta}(\boldsymbol{t})$. Substituting this solution in the remaining equations (31) gives $n_{1}+n_{2}$ constraints of the form

$$
f_{k}(\boldsymbol{t})=0, \quad k=1, \ldots, n_{1}+n_{2} .
$$

It is not difficult to determine the solutions of (31)-(32) when $t_{n}=0, n \geq 4$. Lists of such solutions are given next.

\section{Example: the $H$ model}

Let us consider the $H$ model and assume that $t_{n}=0$ if $n \geq 4$. Then it follows that the condition $\operatorname{det}\left(\partial_{\beta_{i} \beta_{j}} W_{H}(\boldsymbol{t}, \boldsymbol{\beta})\right)=0$ which characterizes $\mathcal{M}_{H}^{\text {sing }}$ reads

$$
32 t_{2}^{2}-24 x t_{3}+72 t_{2} t_{3}\left(\beta_{1}+\beta_{2}\right)+27 t_{3}^{2}\left(\beta_{1}^{2}+6 \beta_{2} \beta_{1}+\beta_{2}^{2}\right)=0 .
$$

There are two generic classes in $\mathcal{M}_{H}^{\text {sing }}$ given by $\mathcal{M}_{H, 1,0}^{\text {sing }}$ and $\mathcal{M}_{H, 0,1}^{\text {sing }}$. Assuming that $\left(t_{1}, t_{2}, t_{3}, \beta_{1}, \beta_{2}\right)$ must be real, both classes include two cases 


$$
\mathcal{M}_{H, 1,0}^{\operatorname{sing}}:\left\{\begin{aligned}
1) \quad x & =\frac{2 t_{2}^{2}-9 \sqrt[3]{6 T^{2}} t_{3}^{4 / 3}}{6 t_{3}}, \\
\beta_{1} & =-\frac{2 t_{2}+\sqrt[3]{36 T} t_{3}^{2 / 3}}{6 t_{3}}, \quad \beta_{2}=\frac{-2 t_{2}+3 \sqrt[3]{36 T} t_{3}^{2 / 3}}{6 t_{3}} . \\
2) \quad x & =\frac{4 t_{2}^{2}+9 \sqrt[3]{6 T^{2}}(1-i \sqrt{3}) t_{3}^{4 / 3}}{12 t_{3}}, \quad \beta_{2}=\frac{-4 t_{2}-3(1+i \sqrt{3}) \sqrt[3]{36 T} t_{3}^{2 / 3}}{12 t_{3}}, \\
\beta_{1} & =\frac{-4 t_{2}+(1+i \sqrt{3}) \sqrt[3]{36 T} t_{3}^{2 / 3}}{12 t_{3}}, \quad
\end{aligned}\right.
$$

where we assume $t_{3}<0$ in the second case.

$$
\left\{\begin{array}{c}
\text { 1) } x=\frac{2 t_{2}^{2}-9 \sqrt[3]{6 T^{2}} t_{3}^{4 / 3}}{6 t_{3}}, \\
\mathcal{M}_{H, 0,1}^{\operatorname{sing}}=\frac{-2 t_{2}+3 \sqrt[3]{36 T} t_{3}^{2 / 3}}{6 t_{3}}, \quad \beta_{2}=-\frac{2 t_{2}+\sqrt[3]{36 T} t_{3}^{2 / 3}}{6 t_{3}}, \\
2) \quad x=\frac{4 t_{2}^{2}+9 \sqrt[3]{6 T^{2}}(1-i \sqrt{3}) t_{3}^{4 / 3}}{12 t_{3}}, \quad \beta_{2}=\frac{-4 t_{2}+(1+i \sqrt{3}) \sqrt[3]{36 T} t_{3}^{2 / 3}}{12 t_{3}}, \\
\beta_{1}=\frac{-4 t_{2}-3(1+i \sqrt{3}) \sqrt[3]{36 T} t_{3}^{2 / 3}}{12 t_{3}}, \quad,
\end{array}\right.
$$

where we assume $t_{3}<0$ in the second case.

\section{Example: the $B$ system}

Let us now consider the system of hodograph equations for the $B$ system with $t_{n}=0$ for all $n \geq 4$. Now the condition $\operatorname{det}\left(\partial_{\beta_{i} \beta_{j}} W_{B}(\boldsymbol{t}, \boldsymbol{\beta})\right)=0$ reduces to

$$
\begin{array}{r}
32 t_{1}^{2}+96 t_{2}\left(\beta_{1}+\beta_{2}\right)+702 t_{3}^{2} \beta_{1}^{2} \beta_{2}^{2}+72\left(3 t_{2}^{2}+t_{1} t_{3}\right) \beta_{1} \beta_{2}+12\left(3 t_{2}^{2}+13 t_{1} t_{3}\right)\left(\beta_{1}^{2}+\beta_{2}^{2}\right)+ \\
486 t_{2} t_{3}\left(\beta_{2} \beta_{1}^{2}+\beta_{2}^{2} \beta_{1}\right)+90 t_{2} t_{3}\left(\beta_{1}^{3}+\beta_{2}^{3}\right)+180 t_{3}^{2}\left(\beta_{2} \beta_{1}^{3}+\beta_{2}^{3} \beta_{1}\right)+45 t_{3}^{2}\left(\beta_{1}^{4}+\beta_{2}^{4}\right)=0 .
\end{array}
$$

There are two classes in $\mathcal{M}_{B, 1,0}^{\text {sing }}$ 
1. $x=\frac{-45 t_{3} t_{2}^{3}+180 t_{1} t_{3}^{2} t_{2}+\sqrt{15}\left(8 t_{1} t_{3}-3 t_{2}^{2}\right) \sqrt{t_{3}^{2}\left(3 t_{2}^{2}-8 t_{1} t_{3}\right)}}{360 t_{3}^{3}}$

$$
\beta_{1}=-\frac{5 t_{2} t_{3}+\sqrt{15} \sqrt{t_{3}^{2}\left(3 t_{2}^{2}-8 t_{1} t_{3}\right)}}{20 t_{3}^{2}}, \quad \beta_{2}=\frac{-3 t_{2} t_{3}+\sqrt{15} \sqrt{t_{3}^{2}\left(3 t_{2}^{2}-8 t_{1} t_{3}\right)}}{12 t_{3}^{2}}
$$

2. $x=\frac{-45 t_{3} t_{2}^{3}+180 t_{1} t_{3}^{2} t_{2}-\sqrt{15}\left(8 t_{1} t_{3}-3 t_{2}^{2}\right) \sqrt{t_{3}^{2}\left(3 t_{2}^{2}-8 t_{1} t_{3}\right)}}{360 t_{3}^{3}}$,

$$
\beta_{1}=\frac{-5 t_{2} t_{3}+\sqrt{15} \sqrt{t_{3}^{2}\left(3 t_{2}^{2}-8 t_{1} t_{3}\right)}}{20 t_{3}^{2}}, \quad \beta_{2}=-\frac{3 t_{2} t_{3}+\sqrt{15} \sqrt{t_{3}^{2}\left(3 t_{2}^{2}-8 t_{1} t_{3}\right)}}{12 t_{3}^{2}}
$$

In the same way it follows that $\mathcal{M}_{B, 0,1}^{\text {sing }}$ has two classes given by

$$
\begin{aligned}
& \text { 1. } x=\frac{-45 t_{3} t_{2}^{3}+180 t_{1} t_{3}^{2} t_{2}-\sqrt{15}\left(8 t_{1} t_{3}-3 t_{2}^{2}\right) \sqrt{t_{3}^{2}\left(3 t_{2}^{2}-8 t_{1} t_{3}\right)}}{360 t_{3}^{3}}, \\
& \beta_{1}=\frac{-\frac{3 t_{2} t_{3}+\sqrt{15} \sqrt{t_{3}^{2}\left(3 t_{2}^{2}-8 t_{1} t_{3}\right)}}{12 t_{3}^{2}}, \quad \beta_{2}=\frac{-5 t_{2} t_{3}+\sqrt{15} \sqrt{t_{3}^{2}\left(3 t_{2}^{2}-8 t_{1} t_{3}\right)}}{20 t_{3}^{2}},}{2 . \quad x=\frac{-45 t_{3} t_{2}^{3}+180 t_{1} t_{3}^{2} t_{2}+\sqrt{15}\left(8 t_{1} t_{3}-3 t_{2}^{2}\right) \sqrt{t_{3}^{2}\left(3 t_{2}^{2}-8 t_{1} t_{3}\right)}}{360 t_{3}^{3}},} \\
& \beta_{1}=\frac{-3 t_{2} t_{3}+\sqrt{15} \sqrt{t_{3}^{2}\left(3 t_{2}^{2}-8 t_{1} t_{3}\right)}}{12 t_{3}^{2}}, \quad \beta_{2}=-\frac{5 t_{2} t_{3}+\sqrt{15} \sqrt{t_{3}^{2}\left(3 t_{2}^{2}-8 t_{1} t_{3}\right)}}{20 t_{3}^{2}} .
\end{aligned}
$$

\section{Acknowledgements}

The authors wish to thank the Spanish Ministerio de Educación y Ciencia (research project FIS2008-00200/FIS) for its finantial support. B. K. is thankful to the Departamento de Física Teórica II for the kind hospitality.

\section{References}

[1] P. Di Francesco, P. Ginsparg and Z. Zinn-Justin, Phys. Rept. 254,1 (1995) 
[2] P. Deift, Orthogonal Polynomials and Random Matrices: A Riemann-Hilbert Approach, Courant Lecture Notes in Mathematics 3, Amer. Math. Soc. Providence, RI, (1999)

[3] L. Martinez Alonso and E. Medina, J. Phys. A: Math. Gen. 40, 14223 (2007)

[4] L. Martinez Alonso and E. Medina, J. Phys. A: Math. Gen. 41, 335202 (2008)

[5] B. A. Dubrovin and S. P. Novikov, Hydrodynamics of weakly deformed soliton lattices. Differential geometry and Hamiltonian theory, Russian Math. Surveys 44, 35 (1989)

[6] C. P. Boyer and J. D. Finley, J. Math. Phys., 23, 1126 (1982)

[7] M. Mineev- Weinstein, P. Wiegman and A. Zabrodin, Phys. Rev. Lett., 84, 5106 (2000)

[8] P. W. Wiegman and P. B. Zabrodin, Comm. Math. Phys., 213, 523 (2000)

[9] I. Krichever, M.Mineev- Weinstein, P. Wiegman and A. Zabrodin, Physica D, 198, 1 (2004)

[10] D. Y. Benney, Stud. Appl. Math. 52 45-50 (1973).

[11] V. E. Zakharov, Func. Anal. Appl. 14, 89 (1980).

[12] B. Dubrovin, T. Grava and C. Klein, J. Nonlinear Science 1957 (2009).

[13] B.G. Konopelchenko, L. Martínez Alonso and E. Medina. Hodograph solutions of the dispersionless coupled KdV hierarchies, critical points and the Euler-Poisson-Darboux equation. arXiv:1003.2892. To appear in J. Phys. A.

[14] Y. Kodama and B.G. Konopelchenko, J. Phys. A: Math. Gen. 35, L489-L500 (2002).

[15] G. Darboux, Lecons sur la theorie general des surfaces II , Gauthier Villars (1915).

[16] V. R. Kudashev and S. E. Sharapov, Phys. Lett. A 154,445 (1991); Theor. Math. Phys. 87, 40 (1991).

[17] F. R. Tian, Duke Math. J. 74203 (1994).

[18] M. V. Pavlov, Hamiltonian formulation of electroforesis equations. Integrable hydrodynamic equations Preprint, Landau Inst. Theor. Phys., Chernogolovsca (1987).

[19] B.G. Konopelchenko and L. Martínez Alonso, J. Phys. A: Math. Gen. 37, 7859 (2004)

[20] I. M. Krichever, Commun. Pure. Appl. Math. 47437 (1994) 